\title{
A Silicon Drift Detector-CMOS front-end system for high resolution X-ray spectroscopy up to room temperature
}

\author{
G. Bertuccio, ${ }^{a, b, 1}$ M. Ahangarianabhari, ${ }^{a, b}$ C. Graziani, ${ }^{a}$ D. Macera, ${ }^{a, b}$ Y. Shi, ${ }^{a, b}$ \\ A. Rachevski, ${ }^{c}$ I. Rashevskaya, ${ }^{c}$ A. Vacchi, ${ }^{c}$ G. Zampa, ${ }^{c}$ N. Zampa,${ }^{c}$ P. Bellutti, ${ }^{d}$ \\ G. Giacomini, ${ }^{d}$ A. Picciotto ${ }^{d}$ and C. Piemonte ${ }^{d}$ \\ ${ }^{a}$ Department of Electronic Engineering, Politecnico di Milano, \\ Via Anzani 42, 22100 Como, Italy \\ ${ }^{b}$ National Institute of Nuclear Physics, INFN Milano, \\ Via Celoria 16, Milano, Italy \\ ${ }^{c}$ National Institute of Nuclear Physics, INFN Trieste, \\ Padriciano, 99, 34149, Trieste, Italy \\ ${ }^{d}$ Fondazione Bruno Kessler, FBK, \\ Via Sommarive 18, I-38123, Trento, Italy \\ E-mail: Giuseppe.Bertuccio@polimi.it
}

\begin{abstract}
A system constituted by a Silicon Drift Detector (SDD), fabricated with an innovative technology for minimizing the anode current, and a new CMOS charge sensitive preamplifier (CSA), designed for ultimate low noise performance, has been realized and experimentally characterized. The SDD is hexagonal with an active area of $13 \mathrm{~mm}^{2}$. The current density measured at the anode with the detector in operating condition is $25 \mathrm{pA} / \mathrm{cm}^{2}$ at $+20^{\circ} \mathrm{C}$. The CSA - named SIRIO - has intrinsic Equivalent Noise Charge (ENC) ranging from 2.9 to 1.5 electrons r.m.s. at $0.8 \mu \mathrm{s}$ and $11 \mu$ s peaking times at room temperature, respectively. With the SDD-SIRIO system at $+21{ }^{\circ} \mathrm{C}$, an energy resolution of $141 \mathrm{eV}$ FWHM on the ${ }^{55} \mathrm{Fe}$ line at $5.9 \mathrm{keV}$ and $74 \mathrm{eV} \mathrm{FWHM}$ on the pulser line with a noise threshold of $170 \mathrm{eV}$ have been measured at $0.8 \mu$ s peaking time. The system has been tested from $-30^{\circ} \mathrm{C}$ to $+30^{\circ} \mathrm{C}$ with energy resolution from $124 \mathrm{eV}$ to $148 \mathrm{eV}$ FWHM at $5.9 \mathrm{keV}$. A moderate cooling at $+10^{\circ} \mathrm{C}$ is sufficient to reach $133 \mathrm{eV} \mathrm{FWHM} \mathrm{at} 5.9 \mathrm{keV}$.
\end{abstract}

KEYWORDS: Solid state detectors; X-ray detectors; Analogue electronic circuits; Front-end electronics for detector readout

${ }^{1}$ Corresponding author.

This is the Accepted Manuscript version of an article accepted for publication in "Journal of Instrumentation". IOP Publishing Ltd is not responsible for any errors or omissions in this version of the manuscript or any version derived from it. The Version of Record is available online at 10.1088/1748-0221/10/01/P01002. 


\section{Introduction}

Silicon Drift Detectors (SDDs), invented in 1984 by Gatti and Rehak, are nowadays widespread devices used in scientific experiments as well as in instruments for industrial applications to detect radiations and particles [1]. In particular the research activity on SDD devoted to X-ray spectroscopy was extremely successful and brought to the development of many commercial high performance detectors thanks to the peculiar feature of low capacitance of SDDs with respect to traditional pn diodes. In SDDs, the electric field - generated by a set of cathodes above the fully depleted bulk - drains electrons on a very small anode (at the center in figure 1 ). The v ery s mall capacitance $(<0.1 \mathrm{pF})$ of the SDD's output electrode is independent of the detector area and brings to multiple advantages to a high resolution X-ray spectroscopy system: a) it reduces the contribution of series voltage noise of the front-end electronics; $b$ ) it shortens the optimum shaping time $\tau_{\text {opt }}$ so that also the contribution of the parallel current noise decreases; c) it allows to operate at higher photon rate because of the shorter $\tau_{\mathrm{opt}}$.

SDDs for X-Ray Spectroscopy with sensitive areas of $10-30 \mathrm{~mm}^{2}$ operate in $0.1-20 \mathrm{keV}$ energy range achieving spectral line widths approaching the Fano limit at working temperature between $-20^{\circ} \mathrm{C}$ and $-50^{\circ} \mathrm{C}$ [2]. The best published values of energy resolution are $123 \mathrm{eV}$ at $-20^{\circ} \mathrm{C}$ [3] and $260 \mathrm{eV}$ at $+25^{\circ} \mathrm{C}$ [4] as FWHM of the $5.9 \mathrm{keV}{ }^{55} \mathrm{Fe}$ line. Nevertheless, the present limit of SDDs and of all semiconductor X-ray detectors still stays on the necessity of cooling in order to achieve high energy resolution, while systems able to operate at room temperature will bring the advantage to avoid bulky, heavy and power consuming cooling devices. The upper limit of the operating temperature is determined by the detector current density, ranging from $1 \mathrm{nA} / \mathrm{cm}^{2}$ down to $200 \mathrm{pA} / \mathrm{cm}^{2}$ at room temperature for typical and best devices, and by the noise of the Front-End Electronics. Both of these factors must be significantly improved in order to operate a 


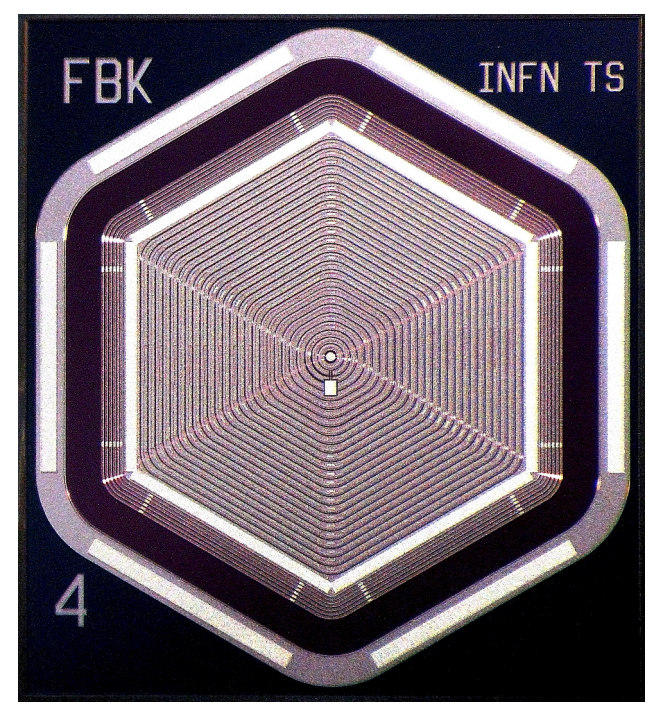

Figure 1. Photo of the Silicon Drift Detector designed for high resolution X-ray spectroscopy. The active area is $13 \mathrm{~mm}^{2}$.

SDD at room temperature without significant d egradation of the e nergy $r$ e solution. O ur research group has worked in both of these directions and the first results of our efforts are presented here.

\section{The system}

\subsection{The drift detector}

The detector has been designed in Trieste and Trento and fabricated at FBK. It is a hexagonal Silicon Drift Detector (SDD) with an active area of $13 \mathrm{~mm}^{2}$. A photo of the detector is shown in figure 1 . T he d etector $\mathrm{h}$ as b een f abricated $\mathrm{u}$ sing a fl oating zo ne, $\mathrm{n}$ - type, $150 \mathrm{~mm}$ diameter, $450 \mu \mathrm{m}$ thick, $\langle 111\rangle$ silicon wafer. A proprietary and innovative technological process has been developed at FBK with the aim of reducing the anode current by minimizing the reverse currents of pn junctions. An integrated resistive voltage divider is present for biasing the drift cathodes.

\subsection{The front-end and pulse processing electronics}

The front-end electronics is constituted by a custom CMOS charge sensitive preamplifier (CSA) — named SIRIO — designed at Politecnico di Milano as an advanced version of a CSA originally designed for $\mathrm{SiC}$ pixel detectors [5]. The preamplifier operates in pulsed reset mode and has been highly optimized to minimize all the noise components [6].

The CSA output signal is filtered using a digital pulse processor (Amptek PX5) performing a triangular pulse shaping with selectable peaking times from $0.8 \mu$ s to $102 \mu \mathrm{s}$ [7].

\section{Experimental}

\subsection{System setup}

The SIRIO preamplifier $\mathrm{h}$ as been mounted in close proximity to the detector anode in order to minimize the stray capacitance, arising from the connection, and reducing the possibility of distur- 


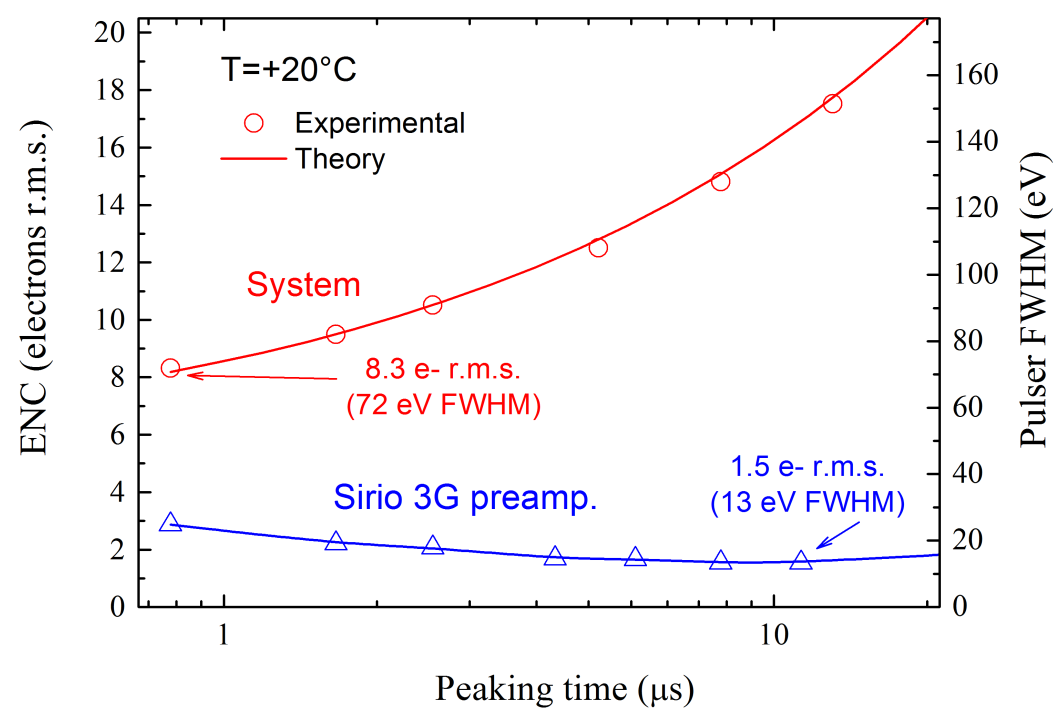

Figure 2. Equivalent Noise Charge of the SIRIO preamplifier a nd of the full S ystem ( SDD+SIRIO) measured at different peaking times of a triangular pulse shaping. The ENC of the SIRIO preamplifier is measured before connecting it to the detector.

bances coupling to the input node. The connection between the SDD and CSA has been made with a wedge bonder with $17 \mu \mathrm{m}$ diameter aluminium wire. A particular care has been devoted to filter the power and voltage supplies for the preamplifier a nd $f$ or $t$ he $d$ e tector. The S DD-CSA system was tested in dry air inside a thermostatic chamber to perform measurements at different operating temperatures, monitored using a thermocouple placed in close proximity to the detector. A pulser (Tektronix AFG3022B) is used to generate the low-noise and highly stable voltage step signals applied to the CSA test capacitance in order to measure the system noise with high precision.

\subsection{Front-end intrinsic noise}

The noise of SIRIO has been measured first with the sy stem in o perating c o ndition but before connecting the preamplifier to the $\mathrm{d}$ e tector. T he E quivalent $\mathrm{N}$ oise $\mathrm{C}$ harge ( $\mathrm{ENC}$ ) $\mathrm{h}$ as b een measured by injecting charge pulses through the test capacitance, whose value has been then precisely determined using a ${ }^{55} \mathrm{Fe}$ spectrum after connecting the detector. Figure 2 shows the results of the measurement of the ENC as function of the peaking time at room temperature $\left(+20^{\circ} \mathrm{C}\right)$. The ENC ranges from 2.9 electrons r.m.s. at $0.8 \mu$ s down to 1.5 electrons r.m.s. at $11 \mu$ s which, at our knowledge, is the lowest noise level ever measured with a CMOS CSA.

\subsection{Detector anode current}

The SDD has been biased to generate a drift field of about $400 \mathrm{~V} / \mathrm{cm}$. From the slope of the preamplifier output voltage, the anode current c an be precisely de termined: $3.3 \mathrm{pA}$ and $12 \mathrm{fA}$ have been measured at $+20^{\circ} \mathrm{C}$ and $-30^{\circ} \mathrm{C}$, respectively. The current density at the anode at room temperature is so $25 \mathrm{pA} / \mathrm{cm}^{2}$, which is the lowest ever measured value on a silicon detector, about an order of magnitude smaller than the best ever published till now. 


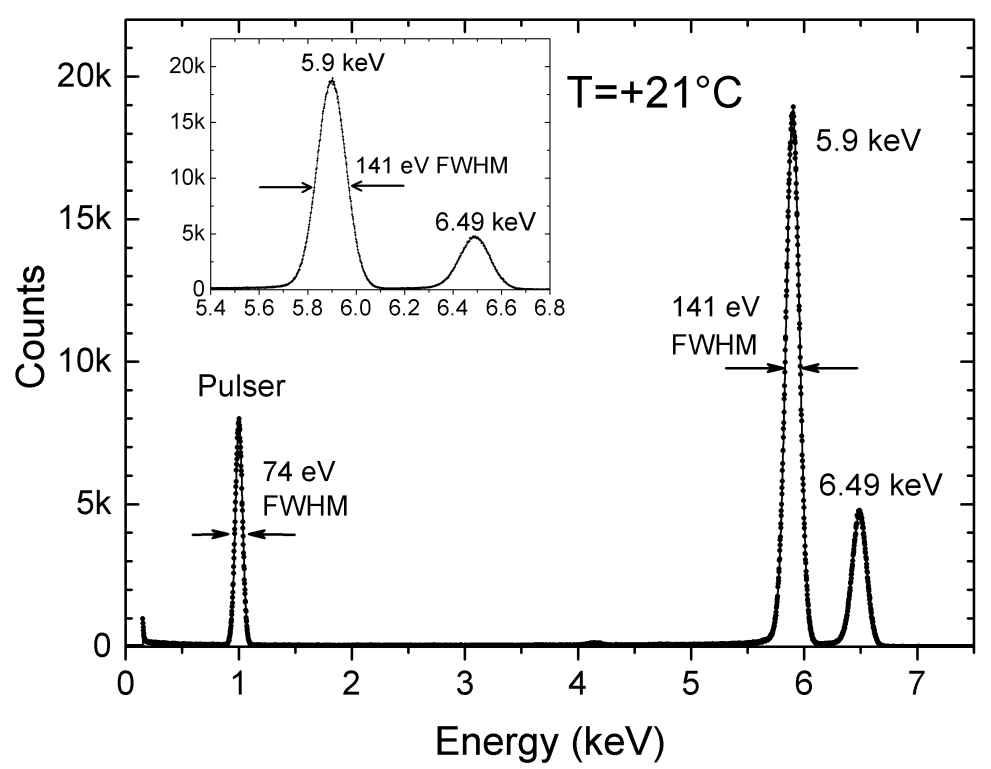

Figure 3. ${ }^{55} \mathrm{Fe}$ spectrum acquired with the system operating at room temperature $\left(+21^{\circ} \mathrm{C}\right)$. The noise threshold is at $170 \mathrm{eV}$.

\subsection{X-ray spectroscopy results}

The performance of the system has been evaluated by irradiating the full detector area with a ${ }^{55} \mathrm{Fe}$ radioactive source with no collimation. Figure 3 shows the best spectrum acquired with the system at $+21^{\circ} \mathrm{C}$ and at $0.8 \mu$ s peaking time. The $5.9 \mathrm{keV}$ line width is $141 \mathrm{eV} \mathrm{FWHM}$ and the pulser line width is $74 \mathrm{eV} \mathrm{FWHM,} \mathrm{corresponding} \mathrm{to} \mathrm{an} \mathrm{ENC}=8.6$ electrons r.m.s. The noise threshold is at $170 \mathrm{eV}$ so that sub- $1 \mathrm{keV}$ spectroscopy is possible with energy resolution of less than $100 \mathrm{eV}$. At the best of our knowledge, this is the highest energy resolution ever achieved at room temperature using a semiconductor detector of such relatively large area $\left(13 \mathrm{~mm}^{2}\right)$.

Figure 2 shows the system ENC measured at different peaking times, showing that the minimum noise is achieved at the minimum available value $t_{\text {peak }}=0.8 \mu \mathrm{s}$. The increasing of ENC with $t_{\text {peak }}$ is in a good agreement with a parallel noise of a $3 \mathrm{pA}$ current (see theoretical curve in figure 2 ), which is very close to the anode current derived from the slope of the preamplifier output voltage. The system has been also tested at different temperatures ranging from $+30^{\circ} \mathrm{C}$ to $-30^{\circ} \mathrm{C}$. Figure 4 shows the FWHM of the $5.9 \mathrm{keV}$ line measured at the optimum peaking times: the line width ranges from $148 \mathrm{eV}$ at $+30^{\circ} \mathrm{C}$ down to $124 \mathrm{eV}$ at $-30^{\circ} \mathrm{C}$. It can be observed that even a moderate cooling at $+10^{\circ} \mathrm{C}$ is sufficient to reach $133 \mathrm{eV} \mathrm{FWHM}$ at $5.9 \mathrm{keV}$.

\section{Conclusions}

The research and development of technological processes aimed to reduce the leakage current of silicon pn junctions allowed the realization of a drift detector with the current density of $25 \mathrm{pA} / \mathrm{cm}^{2}$ at room temperature, the lowest ever measured. At the same time, the research activity on ultralow noise charge sensitive preamplifier brought to the re alization of S IRIO C MOS preamplifier with intrinsic ENC as low as 1.5 electrons r.m.s. It has been presented that the X-ray spectro- 


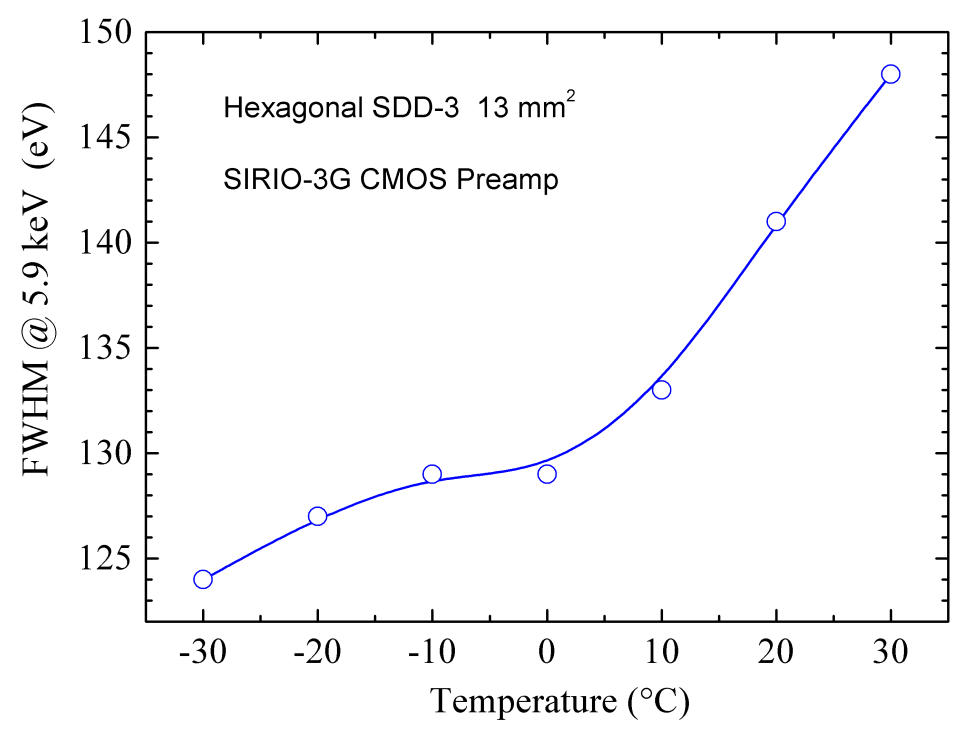

Figure 4. FWHM of the ${ }^{55} \mathrm{Fe}$ line at $5.9 \mathrm{keV}$ measured with the SDD-SIRIO System at different temperatures. The peaking time of triangular shaping is set to minimize the system noise at each temperature.

scopic system SDD-SIRIO operating at room temperature shows intrinsic energy resolutions of $74 \mathrm{eV}$ FWHM (pulser line) $\left(+21{ }^{\circ} \mathrm{C}\right)$, a noise threshold of $170 \mathrm{eV}$ and a $5.9 \mathrm{keV}{ }^{55} \mathrm{Fe}$ line width of $141 \mathrm{eV}$ FWHM. Such achievements open new perspectives for future X-ray spectroscopy systems operating with moderate or even without a cooling apparatus, with significant advantages in terms of compactness and low-power consumption.

\section{Acknowledgments}

The work has been made within the ReDSOX experiment of INFN. G. Bertuccio would like to thanks A.C. Huber and R.H. Redus for the optimized digital pulse processor used in these experiments. Thanks to S. Masci for accurate system assembling and bonding.

\section{References}

[1] E. Gatti and P. Rehak, Review of semiconductor drift detectors, Nucl. Instrum. Meth. A 541 (2005) 47.

[2] R. Redus, A. Huber, J. Pantazis and T. Pantazis, Enhanced energy range thermoelectrically cooled silicon X-ray detectors, IEEE NSS/MIC (2011) 580.

[3] D.M. Schlosser et al., Expanding the detection efficiency of silicon drift detectors, Nucl. Instrum. Meth. A 624 (2010) 270.

[4] L. Bombelli, C. Fiorini, T. Frizzi, R. Alberti and R. Quaglia, High rate X-ray spectroscopy with “CUBE” preamplifier coupled with silicon drift detector, IEEE NSS/MIC (2012) 418.

[5] G. Bertuccio and S. Caccia, Progress in ultra-low-noise ASICs for radiation detectors, Nucl. Instrum. Meth. A 579 (2007) 243.

[6] G. Bertuccio and S.Caccia, Noise minimization of MOSFET input charge amplifiers based on $\Delta \mu$ and $\Delta N$ 1/ fmodels, IEEE Trans. Nucl. Sci. 56 (2009) 1511.

[7] Amptek, Inc., 14 De Angelo Drive, Bedford, MA 01730, U.S.A. 